\title{
Developmental characteristics of the cytokine profile in aqueous humor and its relationship with the inflammatory response in children
}

\author{
Weiyi Lai ${ }^{1 \#}$, Xiaohang Wu ${ }^{1 \#}$, Dongni Wang ${ }^{1}$, Zhenzhen Liu ${ }^{1}$, Duoru Lin ${ }^{1}$, Lanqin Zhao ${ }^{1}$, Weirong Chen ${ }^{1}$, \\ Haotian Lin $^{1,2^{\wedge}}$, Yizhi Liu ${ }^{1}$ \\ ${ }^{1}$ State Key Laboratory of Ophthalmology, Zhongshan Ophthalmic Center, ${ }^{2}$ Center for Precision Medicine, Sun Yat-sen University, Guangzhou, \\ China \\ Contributions: (I) Conception and design: H Lin, Y Liu; (II) Administrative support: H Lin, W Chen, Y Liu; (III) Provision of study materials or \\ patients: W Lai, X Wu, D Wang; (IV) Collection and assembly of data: W Lai, X Wu, D Wang, Z Liu, D Lin; (V) Data analysis and interpretation: \\ W Lai, X Wu, L Zhao; (VI) Manuscript writing: All authors; (VII) Final approval of manuscript: All authors. \\ \#These authors contributed equally to this work. \\ Correspondence to: Prof. Haotian Lin. Zhongshan Ophthalmic Center, Sun Yat-Sen University, 7\# Jinsui Road, Guangzhou, China. \\ Email: linht5@mail.sysu.edu.cn; Prof. Yizhi Liu. State Key Laboratory of Ophthalmology, Zhongshan Ophthalmic Center, Sun Yat-sen University, \\ Guangzhou, China. Email: yizhi_liu@aliyun.com.
}

Background: Although influencing the severity of postoperative intraocular inflammation of congenital cataract, the developmental characteristics of cytokine profile in the aqueous humor during childhood had not been described. And its relationship with the inflammatory response after intraocular surgery remained unsolved.

Methods: Preoperative aqueous humor samples were collected from 65 eyes of congenital cataract patients (CC group) and 13 eyes of age-related cataract patients (ARC group) from January 2015 to May 2017. The concentrations of 22 cytokines were measured. Differences in concentrations between the CC and ARC groups were described. Correlation analysis, least absolute shrinkage and selection operator (LASSO) and multivariate linear regression were then used to investigate the dynamic changes in the cytokine profile with age and their relationships with the postoperative inflammatory response.

Results: The concentrations of granulocyte colony stimulating factor (G-CSF), interferon (IFN)- $\alpha 2$, interleukin (IL)-1 $\alpha$ and IL-7 were higher in the CC group than in the ARC group, while the concentrations of epidermal growth factor (EGF), granulocyte-macrophage colony stimulating factor (GM-CSF), IL-10, IL-12p70, IL-2, IL-3, IL-4, IL-5, IL-6, IL-8, and tumor necrosis factor (TNF)- $\alpha$ were more abundant in the ARC group $(\mathrm{P}<0.05)$. Within the CC group, the concentrations of EGF and IL-3 were positively correlated with age, while negative correlations were observed for monocyte chemotactic protein-1 (MCP-1) and IL-8 $(\mathrm{P}<0.05)$. Further multivariate analysis revealed that age was an independent associated factor of $\mathrm{MCP}-1$ $(\beta=-0.337, \mathrm{P}<0.001)$. IL-3 ( $\beta=-0.490, \mathrm{P}=0.006)$ and $\mathrm{EGF}(\beta=-0.415, \mathrm{P}=0.044)$ were significantly correlated with the postoperative inflammatory response [evaluation of posterior capsule opacification (EPCO)] at 3 and 12 months after surgery, respectively.

Conclusions: This study demonstrated the dynamic change in the cytokine profile of the aqueous humor in children and its relationship with the postoperative inflammatory response. These findings can serve as the foundation for further investigation into the mechanisms that underlie the early development of intraocular immunology.

^ ORCID: 0000-0003-4672-9721. 
Keywords: Aqueous humor; congenital cataract (CC); cytokines; pediatrics

Submitted Sep 04, 2019. Accepted for publication Mar 17, 2020.

doi: 10.21037/atm-19-2377

View this article at: http://dx.doi.org/10.21037/atm-19-2377

\section{Introduction}

A very significant phenomenon exists in clinical practice in which younger children usually suffer from more severe intraocular inflammation than older children, even after the same ophthalmic surgery designed for the same noninflammatory disease. Previous studies have indicated that the blood-ocular barrier (BOB), the intraocular immunosuppressive microenvironment and anterior chamber associated immune deviation (ACAID) contribute to the immune-privileged intraocular environment (1-3). Therefore, the mechanism of the phenomenon in which postoperative intraocular inflammation is usually more severe in younger children may also be explained by an immature $\mathrm{BOB}$ and an underdeveloped immunosuppressive microenvironment or ACAID. Although the ophthalmic immunosuppressive microenvironment and ACAID have been studied carefully from many aspects and have revealed many cytokines contributing to or destroying their mechanisms, such as interleukin (IL)-2, IL-6, IL1RA, IL-10, IL-12, interferon (IFN) $-\gamma$, prostaglandins and RANTES, these research studies were all based on experiments on adult animals and described no developmental characteristics during childhood (3-7).

Previous studies from our research group used human aqueous humor specimens to identify the relationships between cytokine profiles and certain eye diseases, and some evidence of age-related changes was recognized. However, the age-dependent characteristics of the cytokine profiles in the aqueous humor in children remained unknown due to either the limited sample size or the disturbances caused by the inflammatory nature of the studied diseases $(8,9)$.

As one of the leading causes of childhood blindness (10), congenital cataract (CC) is not an inflammatory disease. Therefore, CC can be an ideal model to reflect the developmental characteristics of the cytokine profile in the aqueous humor. The poor prognosis of $\mathrm{CC}$ originates from not only its concealed onset and the difficulties of operative therapy but also the variety of postoperative complications, among which prominent intraocular inflammation is remarkable, especially when intraocular lens (IOL) is implanted in a very young age (11-14). Similarly, for intraocular inflammation, the younger the child is, the more severe the condition after cataract surgery. However, the mechanism underlying this phenomenon is still worth exploring.

Thus, the purpose of this paper is to identify the developmental characteristics of the cytokine profile of the aqueous humor among human children. The findings may help explain the phenomenon we mentioned at the very beginning of this paper and can be the foundation of further research into the details of its underlying mechanism.

\section{Methods}

\section{Subjects and enrollment criteria}

Patients with CC (CC group) or age-related cataract (ARC group) who were undergoing cataract surgery for the first time were prospectively recruited during preoperative screening at the Zhongshan Ophthalmic Center (ZOC), Guangdong, China, from January 2015 to May 2017. The study adhered to the tenets of the Declaration of Helsinki and was approved by the Ethical Review Committee of ZOC. Informed written consent was obtained from all patients in the ARC group and from at least one guardian of each participating child in the CC group.

The eligibility criteria for the CC group were as follows: (I) patients with bilateral or unilateral CC; (II) patients less than 10 years old; (III) patients for whom informed, written consent was provided by at least one guardian; and (IV) patients who were able to participate in the postsurgical follow-up within at least 3 months.

The eligible criteria of the ARC group were as follows: (I) patients with ARC; (II) 60-75 years old; and (III) informed, written consent from the patient himself.

Although CC is not an inflammatory disease, we excluded patients if they met any one of the following criteria to reflect the physiologic intraocular environment to the utmost extent: (I) pre- or postmature birth; (II) systemic disorders or diseases; (III) history of allergy; (IV) history of surgery, trauma and other eye diseases; and (V) traumatic cataract, metabolic cataract, radioactive cataract, drug-induced cataract, or cataract induced 
by infectious disease, myopia or any other evident causes.

Demographic information, including age, gender, laterality and specific surgery procedures after aqueous humor sampled, was obtained from each enrolled patient. All subjects underwent a thorough ophthalmic evaluation, including slit lamp examination, fundus photography and B-scanning. All patients in the ARC group were treated by cataract phacoemulsification and IOL implantation (Phaco + IOL). For the CC group, personalized procedures were designed for each patient. In general, the procedures for CC include the following: (I) lens aspiration without IOL implantation (I/A); (II) lens aspiration with IOL implantation (I/A + IOL); (III) lens aspiration with posterior continuous curvilinear capsulorhexis and anterior vitrectomy (I/A + PCCC + A-Vit); and (IV) lens aspiration with IOL implantation, posterior continuous curvilinear capsulorhexis and anterior vitrectomy $(\mathrm{I} / \mathrm{A}+\mathrm{IOL}+\mathrm{PCCC}+\mathrm{A}-\mathrm{Vit})$.

\section{Aqueous humor collection}

Approximately $200 \mu \mathrm{L}$ of aqueous humor was collected from one eye of each enrolled patient prior to any surgical procedure. Via a limbus paracentesis, a 30-gauge needle on a tuberculin micro syringe was used to aspirate aqueous humor from the anterior chamber. All samples were obtained before any conjunctival or intraocular manipulation to avoid breakdown of the blood-aqueous barrier associated with surgical trauma. The aqueous humor samples were stored immediately under $-80^{\circ} \mathrm{C}$ until analysis. Specifically, all surgeries and sample collections involved were performed by the same expert surgeon.

\section{Measurement of cytokines}

The concentrations of cytokines were analyzed by using a multiplex bead immunoassay (Milliplex Human Cytokine kit; Millipore Corp., Billerica, MA, USA), compatible instrument and software (MILLIPLEX map Multiplex Detection based on Luminex platform, Milliplex Analyte 5.1, Millipore Corp., Billerica, MA, USA). A 25- $\mu \mathrm{L}$ volume of aqueous humor sample was used in each reaction. From the information provided by the manufacturer, the multiplex assay kit can quantitatively measure multiple cytokines from as little as $25 \mu \mathrm{L}$ of bodily fluids. Each sample was measured in duplicate. And according to the manufacturer, only measurements with the coefficient of variation lower than $15 \%$ would be retained for further analysis.

The following factors related to the vast majority of inflammatory and proliferative processes were analyzed simultaneously: (I) the cytokines IL- $1 \alpha$, IL- $1 \beta$, IL1RA, IL-2, IL-3, IL-4, IL-5, IL-6, IL-7, IL-10, IL$12 \mathrm{p} 40$, IL-12p70, and IL-13; (II) the cytokines tumor necrosis factor (TNF)- $\alpha$, IFN- $\gamma$, and IFN- $\alpha 2$; (III) the chemokines granulocyte colony stimulating factor (G-CSF), granulocyte-macrophage colony stimulating factor (GMCSF), monocyte chemotactic protein (MCP)-1/CCL2, and IL-8/CXCL8; and (IV) the growth factors epidermal growth factor (EGF) and fibroblast growth factor (FGF-2).

\section{Assessment of inflammatory proliferative response after surgery}

The inflammatory proliferative response after cataract surgery in the CC group was defined and assessed by evaluating posterior capsule opacification scores (EPCO scores) at 3/6/12 months after cataract surgery. The EPCO score was evaluated via slit lamp biomicroscopic examination (15). Pupils were dilated with tropicamide $1 \%$ and phenylephrine $2.5 \%$. Digital retroillumination photographs were taken with a slit lamp equipped with a digital photo adapter (BX 900H Photo Slit Lamp, HaagStreit AG, Switzerland) and a high-resolution digital camera. The Evaluation of Posterior Capsular Opacification system (EPCO2000, developed by Tetz MR and associates, Berlin, Germany) was used to assess the EPCO scores (16). In this system, the amount of opacification within the pupil area is graded clinically from 0 to 4 (Table 1). A total posterior capsule opacification (PCO) score is calculated by multiplying the opacification density score by the percentage of the capsule area that is opacified (Figure S1).

\section{Statistical analysis}

The Shapiro-Wilk test was used to evaluate the normality of distribution for every variable. Two-tailed $t$-test for independent samples was used to define the differences in cytokine concentrations between the CC and ARC groups if the variables were normally distributed $(\mathrm{P} \geq 0.05$ in the ShapiroWilk test). Otherwise, the Mann-Whitney $U$ test was used. Within the CC group, the concentrations of cytokines were correlated with each other and age by performing Pearson correlation if they were normally distributed. The Spearman correlation was used in other cases.

For every cytokine or EPCO score as a dependent variable, any other cytokines or age correlated with it would be enrolled in the least absolute shrinkage and selection 
Table 1 EPCO grading scale

\begin{tabular}{ll}
\hline Grade & Description \\
\hline 1 & Minimum wrinkling of capsule with a fine layer of LECs \\
2 & Mild honeycomb PCO; thicker layer of LECs with dense fibrosis \\
3 & Classic elschnig pearls; very thick layer of LECs \\
4 & Severe opacity with a darkening effect \\
\hline
\end{tabular}

EPCO, evaluation of posterior capsule opacification; LECs, lens epithelial cells; PCO, posterior capsule opacification.

Table 2 Patient baseline characteristics

\begin{tabular}{lcc}
\hline Variable & Congenital cataract & Age-related cataract \\
\hline Sample size & 65 & 13 \\
Sex ratio (male\%) & $58.46 \%(38 / 65)$ & $46.15 \%(6 / 13)$ \\
Ratio of bilaterality & $72.31 \%(47 / 65)$ & $100.00 \%(13 / 13)$ \\
Age when sampled (mean \pm SD, years) & $3.89 \pm 2.34$ & $70.46 \pm 3.93$ \\
Major surgical procedures & $\mathrm{I} / \mathrm{A}+\mathrm{IOL}+\mathrm{PCCC}+\mathrm{A}-\mathrm{Vit}^{\dagger}(84.62 \%, 55 / 65)$ & ${\mathrm{Phaco}+\mathrm{IOL}^{\ddagger}(100.00 \%, 13 / 13)}$ \\
\hline
\end{tabular}

Age distribution of the congenital cataract group: 7 patients ( $\leq 1$ years old); 8 patients (1-2 years old); 10 patients (2-3 years old); 13 patients (3-4 years old); 11 patients (4-5 years old); 5 patients $\left(5-6\right.$ years old); 11 patients (>6 years old). ${ }^{\dagger}, \mathrm{I} / \mathrm{A}+\mathrm{IOL}+\mathrm{PCCC}+\mathrm{A}-\mathrm{Vit}=$ lens aspiration with IOL implantation, posterior continuous curvilinear capsulorhexis and anterior vitrectomy; ${ }^{\ddagger}, \mathrm{Phaco}+\mathrm{IOL}=\mathrm{cataract}$ phacoemulsification and intraocular lens implantation.

operator model (LASSO model) to solve the problem of multicollinearity and to prescreen for the independent associated factors. The rest of the correlated variables after being filtered by the LASSO model were further analyzed by multivariate linear regression to evaluate the true effect of each independent predictor on the dependent variable. Backward selection was used in the process of multivariate linear regression if possible. Standardized coefficient ( $\beta$ ), P value of significance of every predictor, adjusted $\mathrm{R}$ square and $\mathrm{P}$ value of regression were recorded. $\mathrm{P}$ values less than 0.05 were considered statistically significant in this study.

The data were processed and analyzed statistically by using IBM SPSS statistical software (version 22.0, SPSS Inc., Chicago, IL, USA) and the glmnet (17) package of $\mathrm{R}$ (version 3.4.1, R Foundation for Statistical Computing, Vienna, Austria). Power analysis was carried out by using G*Power (version 3.1.9.4, developed by Franz Faul and associates, Kiel University, Germany) (18).

\section{Results}

\section{Demographic and clinical characteristics of the patients}

A total of 65 patients were enrolled into the CC group, while 13 patients were enrolled into the ARC group. Only one eye of each patient was sampled in this study, regardless of the unilaterality or bilaterality of their cataract. If the patient underwent bilateral cataract surgeries, we collected the aqueous humor sample from the first surgical eye only. The demographic and clinical data of the included subjects are summarized in Table 2. Both the CC and ARC groups consisted mostly of bilateral patients $(72.31 \%$ in the CC group; $100 \%$ in the ARC group). A difference in age distribution could be expected $(3.89 \pm 2.34$ years old in the CC group; 70.46 \pm 3.93 years old in the ARC group). Within the CC group, the age distribution was relatively balanced, with more than 5 patients in every 1 -year interval. With respect to the surgical procedures, I/A + IOL + PCCC + A-Vit and Phaco + IOL were performed in the majority of patients in the CC group $(84.62 \%, 55 / 65)$ and the ARC group $(100 \%, 13 / 13)$, respectively.

\section{Differences in the cytokine microenvironment between the two groups}

All 22 cytokines on the panel were detected in all 78 aqueous samples. The detailed concentrations and significant differences of these cytokines are summarized 
Table 3 Comparison of cytokine microenvironments between adults and children

\begin{tabular}{|c|c|c|c|c|c|c|c|}
\hline Factors & \multicolumn{3}{|c|}{ Congenital cataract $(n=65)$} & \multicolumn{3}{|c|}{ Age-related cataract $(n=13)$} & $\begin{array}{c}\text { P (Mann- } \\
\text { Whitney U } \\
\text { test) }\end{array}$ \\
\hline EGF & $3.19 \pm 0.95$ & 3.01 (2.68-3.39) & $<0.001$ & $3.39 \pm 0.41$ & 3.39 (3.23-3.69) & 0.051 & $0.013^{*}$ \\
\hline FGF-2 & $15.48 \pm 30.40$ & 6.47 (1.28-19.57) & $<0.001$ & $17.96 \pm 23.33$ & $11.56(6.47-16.95)$ & $<0.001$ & 0.175 \\
\hline GM-CSF & $1.26 \pm 0.28$ & $1.22(1.07-1.40)$ & $<0.001$ & $1.36 \pm 0.13$ & $1.40(1.24-1.48)$ & 0.733 & $0.020^{*}$ \\
\hline IFN- $\alpha 2$ & $7.25 \pm 7.23$ & $3.92(2.65-8.94)$ & $<0.001$ & $1.92 \pm 0.25$ & $1.93(1.75-2.12)$ & 0.421 & $<0.001^{\star \star}$ \\
\hline IL-10 & $1.28 \pm 0.48$ & $1.20(1.09-1.36)$ & $<0.001$ & $1.41 \pm 0.28$ & $1.36(1.24-1.51)$ & 0.001 & $0.013^{*}$ \\
\hline IL-12p40 & $2.29 \pm 1.56$ & $1.84(1.44-2.27)$ & $<0.001$ & $1.77 \pm 0.23$ & $1.69(1.55-1.92)$ & 0.078 & 0.605 \\
\hline IL-1RA & $10.76 \pm 30.85$ & $1.85(1.02-5.91)$ & $<0.001$ & $1.44 \pm 0.46$ & $1.37(1.09-1.83)$ & 0.806 & 0.091 \\
\hline IL-1 $1 \alpha$ & $1.21 \pm 0.44$ & $1.12(0.98-1.29)$ & $<0.001$ & $1.00 \pm 0.12$ & $0.98(0.92-1.09)$ & 0.342 & $0.016^{*}$ \\
\hline IL-1 $\beta$ & $0.66 \pm 0.13$ & $0.63(0.55-0.73)$ & $<0.001$ & $0.70 \pm 0.07$ & $0.73(0.63-0.76)$ & 0.268 & 0.064 \\
\hline IL-2 & $1.47 \pm 0.29$ & $1.44(1.33-1.60)$ & $<0.001$ & $1.69 \pm 0.14$ & $1.63(1.56-1.82)$ & 0.275 & $<0.001^{\star \star}$ \\
\hline IL-3 & $0.51 \pm 0.58$ & $0.16(0.01-1.05)$ & $<0.001$ & $1.02 \pm 0.10$ & $1.00(0.96-1.10)$ & 0.941 & $0.012^{*}$ \\
\hline IL-4 & $2.39 \pm 1.38$ & $1.97(1.65-2.80)$ & $<0.001$ & $3.40 \pm 0.77$ & 3.39 (2.80-4.10) & 0.935 & $<0.001^{\star \star}$ \\
\hline IL-5 & $0.59 \pm 0.98$ & $0.43(0.36-0.57)$ & $<0.001$ & $0.58 \pm 0.04$ & $0.58(0.55-0.62)$ & 0.441 & $0.002^{\star \star}$ \\
\hline $\mathrm{IFN}-\gamma$ & $1.30 \pm 0.26$ & $1.24(1.15-1.43)$ & $<0.001$ & $1.30 \pm 0.09$ & $1.26(1.24-1.37)$ & 0.792 & 0.455 \\
\hline
\end{tabular}

*, correlation is significant at the 0.05 level (2-tailed); ${ }^{* *}$, correlation is significant at the 0.01 level (two-tailed). SD, standard deviation; EGF, epidermal growth factor; FGF, fibroblast growth factor; G-CSF, granulocyte colony stimulating factor; GM-CSF, granulocyte-macrophage colony stimulating factor; IFN, interferon; IL, interleukin; MCP-1, monocyte chemotactic protein-1; TNF, tumor necrosis factor.

in Table 3. Since the cytokines of the CC group were not normally distributed according to the ShapiroWilk test, the Mann-Whitney $\mathrm{U}$ test was chosen for the comparison. Significant differences were noted in most of the cytokines between the two groups. The aqueous humor concentrations of G-CSF, IFN- $\alpha 2$, IL- $1 \alpha$ and IL-7 were higher in the CC group, while EGF, GM-CSF, IL-10, IL12p70, IL-2, IL-3, IL-4, IL-5, IL-6, IL-8, and TNF- $\alpha$ were more abundant in the ARC group (all $\mathrm{P}<0.05$ ). According to these findings, the cytokine microenvironment of the aqueous humor is indeed very different between adults and children.

\section{Correlation analysis between age and cytokines during childbood}

Since a distinct cytokine profile was found in the CC group, we further investigated if there was any trend of development during childhood. In addition, the cytokines could form a regulatory network for each other. Therefore, 

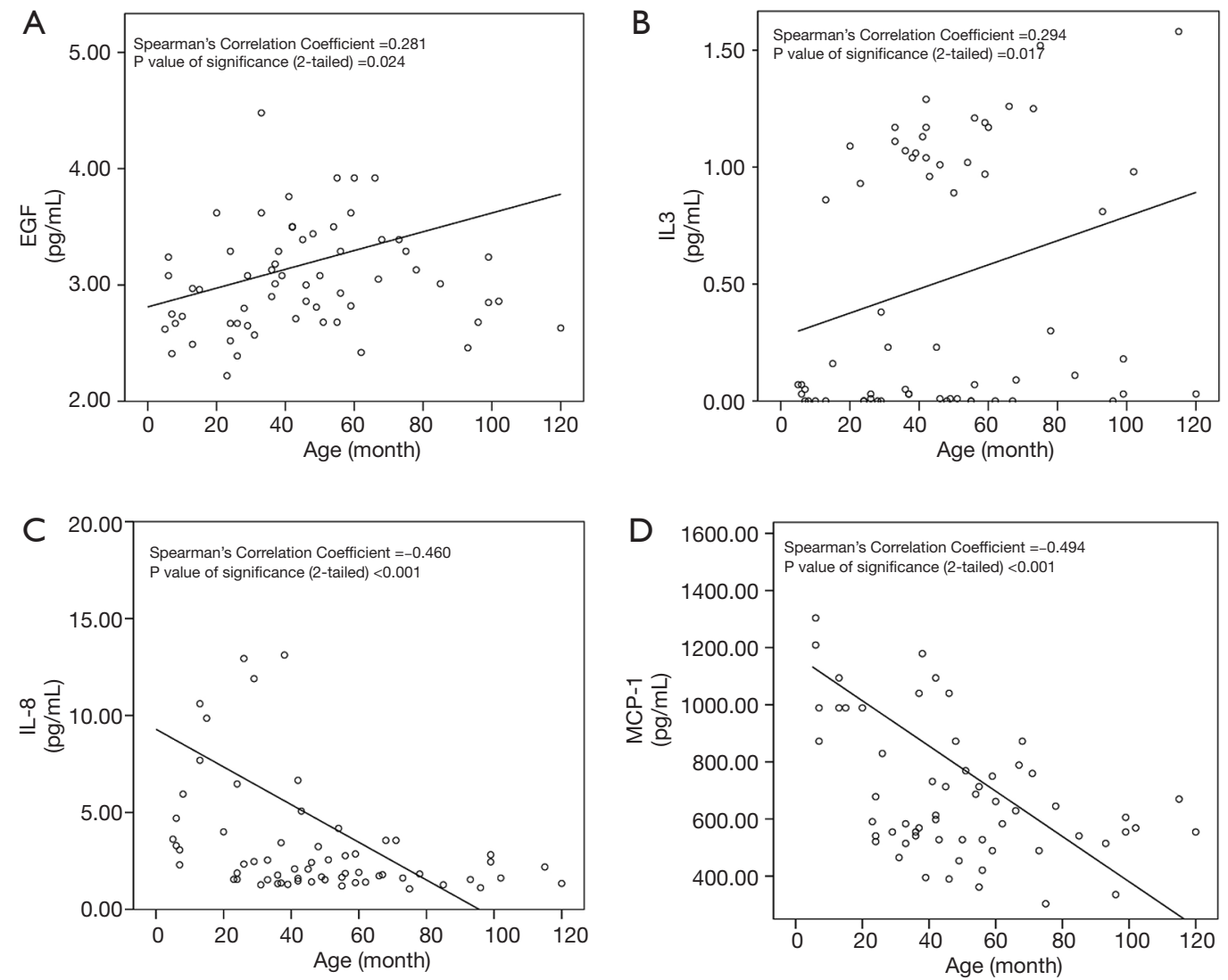

Figure 1 Four age-correlated cytokines. (A) EGF; (B) IL-3; (C) IL-8; (D) MCP-1. EGF, epidermal growth factor; MCP-1, monocyte chemotactic protein-1; IL, interleukin.

all cytokines and age were included in the correlation analysis. Spearman correlation was performed because of the nonnormality of children's cytokine concentrations. The detailed information of the correlation analysis is presented in Table S1.

Four of the 22 cytokines were significantly correlated with age. The concentrations of EGF $(\rho=0.281, \mathrm{P}=0.024)$ and IL-3 $(\rho=0.294, P=0.017)$ were positively correlated with age, while those of MCP-1 $(\rho=-0.494, \mathrm{P}<0.001)$ and IL-8 $(\rho=-0.460, \mathrm{P}<0.001)$ were negatively correlated (Figure 1). However, most cytokines were also significantly correlated with other cytokines, which may indicate a regulatory network among themselves. For example, EGF was significantly correlated with FGF-2, GM-CSF, IL-10, IL-12p70, IL-13, IL-1 $\beta$, IL-3, IL-4, IL-5, IL-6, TNF- $\alpha$ and age simultaneously. Therefore, multivariate analysis was performed following the correlation analysis to confirm the true independent correlation of age growth and the regulatory network. Power analysis revealed that the sensitivity of correlation detection was 0.34 [given $\alpha$ err prob $=0.05$, power $(1-\beta$ err prob $)=0.8$, total sample size $=65]$.

\section{Multivariate analysis based on correlations}

Since the independent effect of age growth on cytokine concentrations was not definite in the univariate analysis above, we further analyzed every dependent cytokine with LASSO regression and multivariate linear regression to screen for the true independent correlations. The results of the four cytokines correlated with age are listed in Table 4, while the other cytokines are summarized in Table S2.

Among the four age-correlated cytokines, the independent correlation of age growth remained significant for MCP-1 only ( $\beta=-0.337, \mathrm{P}<0.001$, adjusted $\mathrm{R}$ square $=0.542)$. Other cytokines were mostly correlated with their fellow cytokines but not age growth independently according to the findings of multivariate analysis (adjusted $\mathrm{R}$ square $>0.5$ ), which further implied the existence of a selfbalanced microenvironment. For example, although EGF was significantly correlated with eleven other cytokines and 
Table 4 Univariate and multivariate analyses of four age-correlated cytokines

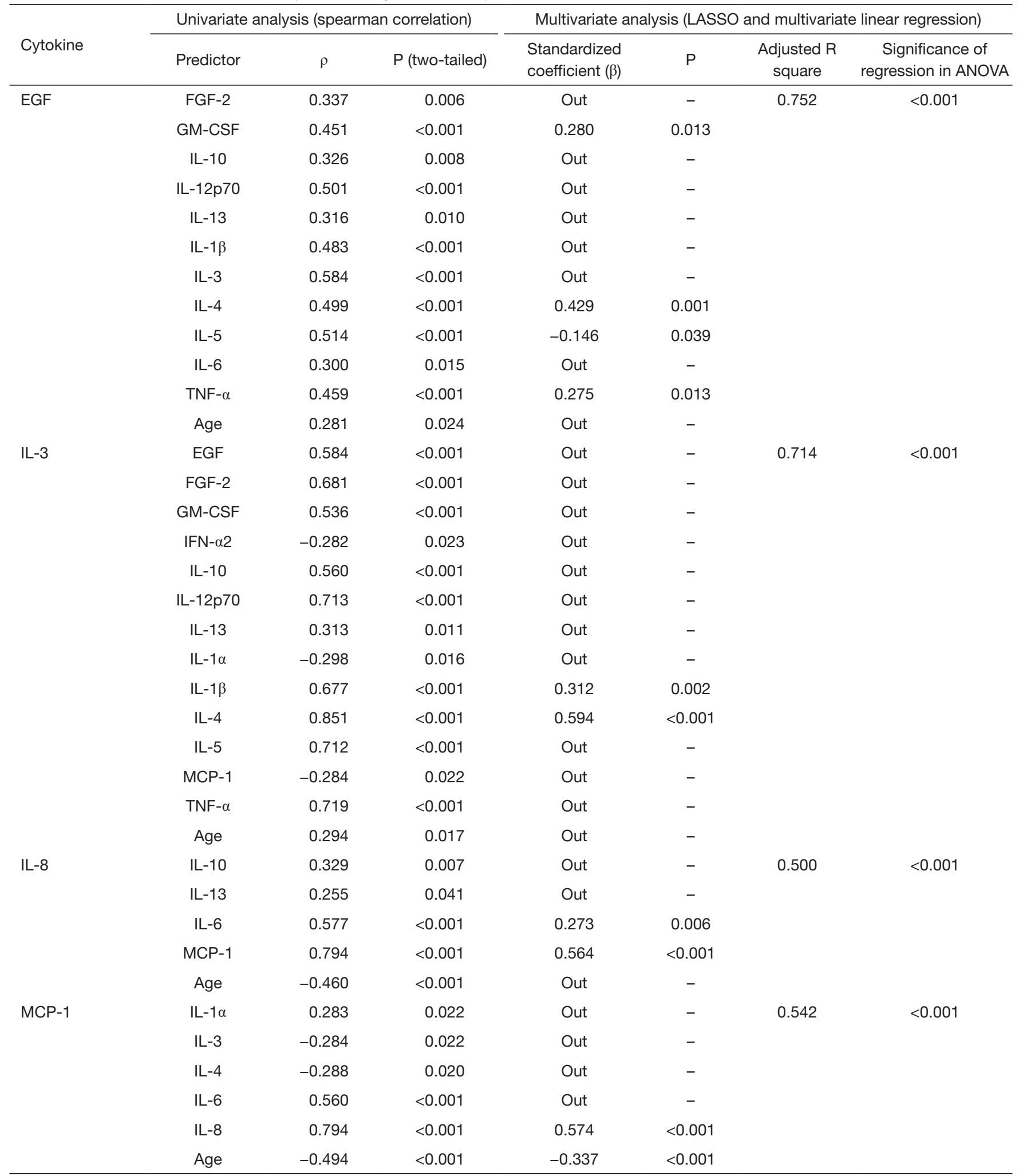

LASSO, least absolute shrinkage and selection operator; EGF, epidermal growth factor; FGF, fibroblast growth factor; GM-CSF, granulocyte-macrophage colony stimulating factor; IL, interleukin; TNF, tumor necrosis factor; IFN, interferon; MCP-1, monocyte chemotactic protein-1. 
Table 5 Univariate and multivariate analyses of the inflammatory proliferative response after surgery

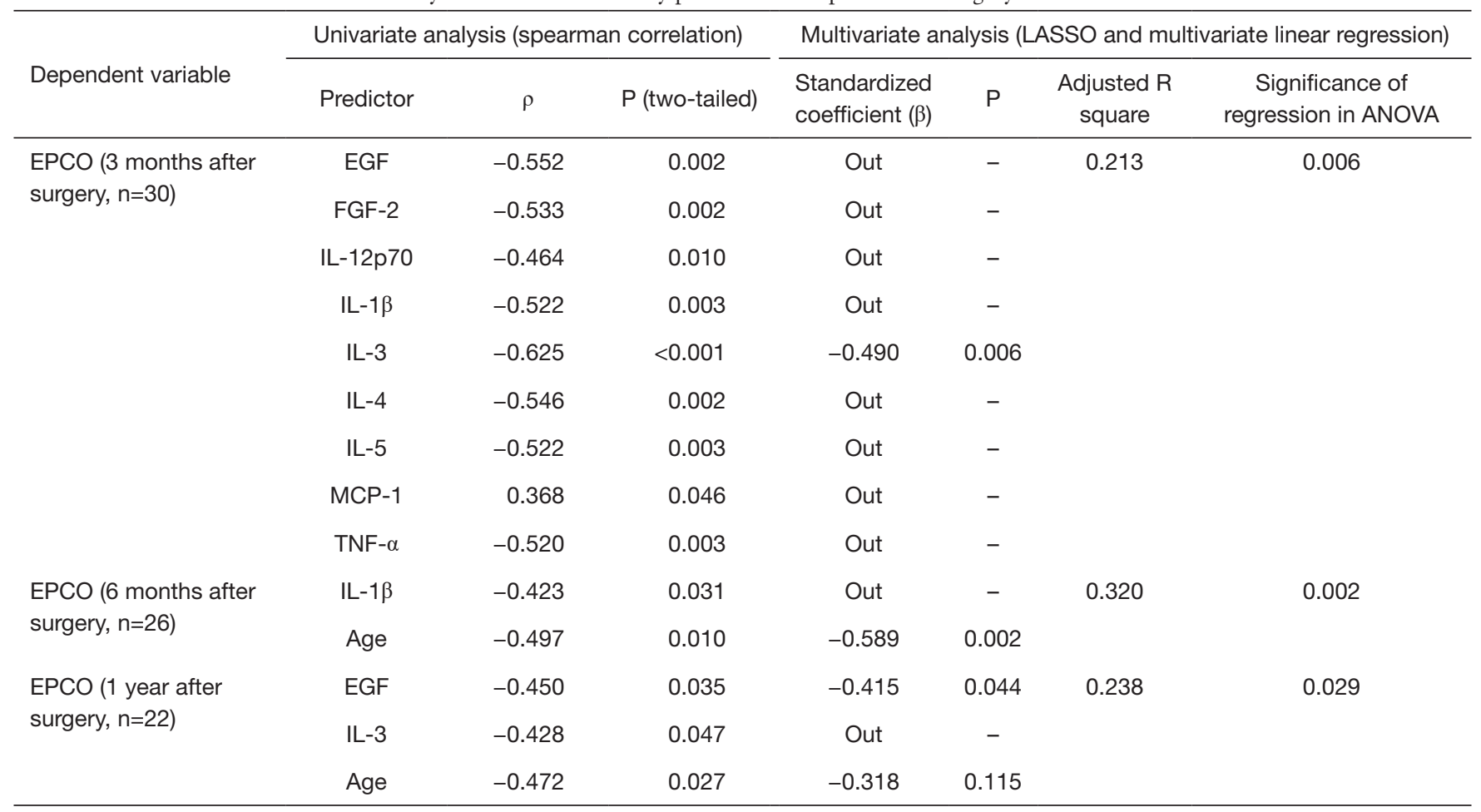

LASSO, least absolute shrinkage and selection operator; EPCO, evaluation of posterior capsule opacification; EGF, epidermal growth factor; FGF, fibroblast growth factor; IL, interleukin; MCP-1, monocyte chemotactic protein-1; TNF, tumor necrosis factor.

age growth simultaneously, it was largely predicted by only IL-4, IL- 5 and TNF- $\alpha$ after the screening of LASSO and multivariate linear regression (adjusted $\mathrm{R}$ square $=0.752$, $\mathrm{P}<0.001)$.

\section{Postoperative inflammatory proliferation and preoperative microenvironment}

To determine if there is any influence of age growth and preoperative cytokine profile on the postoperative inflammatory response during childhood, we subjected the EPCO scores at 3 months, 6 months and 1 year after cataract surgery to univariate and multivariate analyses. The results of Spearman correlation analysis and multivariate linear regression are listed in Table 5. To exclude the influence of different surgical procedures, only patients who underwent I/A + IOL + PCCC + A-Vit $(84.62 \%, 55 / 65$ in CC group) were included in this part of the analysis.

The 3-month postoperative EPCO score was negatively correlated with EGF, FGF-2, IL-12p70, IL$1 \beta$, IL-3, IL-4, IL-5 and TNF- $\alpha$ and was positively correlated with MCP-1 (all $\mathrm{P}<0.05$ ). However, only IL-3 was left after LASSO screening and variable selection of multivariate linear regression $(\beta=-0.490, \mathrm{P}=0.006)$. Regarding the EPCO score 6 months after surgery, it was negatively correlated with IL- $1 \beta$ and age growth (all $\mathrm{P}<0.05)$. Age growth reduced the 6 -month postoperative EPCO score in multivariate linear regression $(\beta=-0.589$, $\mathrm{P}=0.002)$. At 1 year after surgery, the EPCO score was negatively correlated with EGF, IL-3 and age growth (all $\mathrm{P}<0.05)$. Only EGF remained significant in multivariate analysis, which indicated that it weakened the postoperative proliferation of lens epithelial cells $(\beta=-0.415, \mathrm{P}=0.044)$.

\section{Conclusions}

Although it is commonly recognized that the inflammatory response is more severe in younger children even after the same intraocular surgery, the developmental mechanism underlying this phenomenon remains a mystery. Our study found some important inflammatory factors whose concentrations in aqueous humor changes during infancy and early childhood, such as IL-8 and MCP-1. We also depicted some of their correlations with postoperative 
intraocular inflammation after cataract surgery.

Several other studies had revealed that the concentrations of aqueous cytokines were different between children and adults in some inflammatory disorders $(19,20)$. It's clear that they cannot be compared to CC, a noninflammatory ocular disease. But even if we compared CC with ARC in our study, the imbalance was still obvious. Previous study by Zheng et al. have shown higher aqueous levels of IFN- $\gamma$, IL-13, IL-12p70, CCL3, VEGF, and their positive correlations with age in the ARC group (21). Their findings partially verified ours regarding the comparisons between $\mathrm{CC}$ and $\mathrm{ARC}$, and demonstrated the distinctiveness of cytokine profile in early childhood. But trends of developments in the aqueous cytokine profile during early childhood were not reported in their article, because of the limited sample size (12 CC patients) and narrow age span (3-6.5 years old).

Besides, some studies also recruited CC patients into control group when they highlighted the characteristics of cytokine profiles in the aqueous humor of other pediatric eye diseases. Higher levels of TGF- $\beta 1$, survivin, IL-6, IL-7, IL-8 and EGF were detected in the aqueous humor of patients with retinoblastoma $(22,23)$. And TGF- $\beta 2$ was found related with congenital ectopia lentis (24). These findings indicated that aqueous cytokine profiles could reflect the pathologic developments of ocular tissues, and generated inspirations that it may also reflect physiologic development during childhood. However, the age spans and sample sizes of their CC groups were not suitable to further explore the subject.

Researchers had also investigated the relationships between aqueous cytokines and postoperative changes of CC. For example, the level of aqueous platelet-derived growth factor (PDGF) was found negatively correlated with postoperative axial elongation but positively correlated with the severity of PCO, while IL-1RA negatively correlated with PCO $(9,25)$. Since PDGF promotes wound healing and collagen synthesis, and IL-1RA shows anti-inflammatory instincts in many other occasions, the correlations can be explained to a certain degree. Although either different cytokines or different outcomes were measured, their findings contributed to more comprehensive understandings of this topic. It should also be noticed that different age spans, sample sizes or sampling techniques can lead to different measured values, due to the rapid development and significant inter-individual difference in the aqueous environment of young children. Thus, further research in this field should look more into these influencing factors.
With a mean age of $3.89 \pm 2.34$ years, our samples represent different period of childhood. We revealed several age-correlated cytokines in childhood, i.e., concentrations of EGF and IL-3 increased with age, while those of MCP1 and IL-8 decreased with age. Furthermore, age was an independent associated factor of MCP-1 in the multivariate linear regression.

It is generally recognized that MCP-1 is a chemoattractant for basophils, B cells, dendritic cells, monocytes, natural killer (NK) cells, and activated $\mathrm{T}$ cells and that IL-8 is a chemotactic and proadhesive factor for neutrophils, T cells, basophils, and eosinophils and activates neutrophils and monocytes (26). A previous study focused on the joint increase of MCP-1 and IL- 8 in the aqueous humor after cataract surgery in old people also indicated the important roles of both cytokines in the postoperative inflammatory response (27). Therefore, the decreasing trends of both MCP-1 and IL-8 in childhood may partly explain the usually milder inflammatory response in elder children after cataract surgery. We also saw evidence of a regulatory network between cytokines in the aqueous humor of children since the concentrations of many cytokines could be largely predicted by two to five other cytokines using multivariate linear regression (see the details in Table 4, Table S2).

Moreover, the postoperative inflammatory proliferation levels of lens epithelial cells, which was measured by EPCO score in our study, were correlated with the aqueous cytokines. Among them, IL-3 and EGF were prominent since they remained significant in the multivariate analysis 3 month and 1 year after surgery, respectively (Table 5). Since we found that they increased with age in a previous analysis, age growth may alleviate inflammatory proliferation through EGF and IL-3. However, both EGF and IL-3 seemed to weaken proliferation according to the statistical analysis, which was in contradiction with their traditional functions (26). This is probably a consequence of the special inflammatory environment or the dramatic changes in their concentrations after surgery.

There were some study limitations. First, the sample size for postsurgical follow-up was limited, which may result in an underpowered conclusion regarding postoperative inflammatory proliferation. Second, our specimens could not fully represent healthy aqueous humor, and any generalization from our findings should be very cautious. Finally, it is not suitable to study the detailed regulatory mechanism and postoperative changes of the cytokine profile in clinical circumstances. Although the postoperative 
aqueous humor specimens can be helpful in explaining the postoperative response, they are very hard to collect in clinical scenarios, especially when children are involved.

In conclusion, we described the cytokine profile of the aqueous humor from human children and found some age-dependent characteristics. In childhood, MCP-1 and IL-8 decreased with age, while EGF and IL-3 increased with age. These trends could exert an influence on the inflammatory response after intraocular surgery. However, further research into the detailed regulatory mechanism is still needed.

\section{Acknowledgments}

Funding: This work was supported by the National Key R\&D Program of China (2018YFC0116500); the Key Research Plan for the National Natural Science Foundation of China in Cultivation Project (91846109); the National Natural Science Foundation of China-General Programs (81770967, 81873675); National Natural Science Fund for Distinguished Young Scholars (81822010); the National Natural Science Foundation -Young Scientists Fund (81800810); the Science and Technology Planning Projects of Guangdong Province (2018A030310104, 2018B010109008, 2019B030316012).

\section{Footnote}

Provenance and Peer Review: This article was commissioned by the Guest Editor (Dr. Andrzej Grzybowski) for the series "Recent developments in cataract surgery" published in Annals of Translational Medicine. The article was sent for external peer review organized by the Guest Editor and the editorial office.

Conflicts of Interest: All authors have completed the ICMJE uniform disclosure form (available at http:// dx.doi.org/10.21037/atm-19-2377). The series "Recent developments in cataract surgery" was commissioned by the editorial office without any funding or sponsorship. The authors report grants from National Key R\&D Program of China, grants from National Natural Science Foundation of China, grants from the Science and Technology Planning Projects of Guangdong Province, during the conduct of the study. The authors have no other conflicts of interest to declare.

Ethical Statement: The authors are accountable for all aspects of the work in ensuring that questions related to the accuracy or integrity of any part of the work are appropriately investigated and resolved. All procedures performed in studies involving human participants were in accordance with the ethical standards of the institutional research committee (Ethical Review Committee of ZOC, reference number: 2013PRLL001) and with the 1964 Helsinki declaration and its later amendments or comparable ethical standards. Informed consent was obtained from all adult participants included in the study. As for participating child, informed consent was obtained from at least one guardian of each. And the study won't affect the management of the patients.

Open Access Statement: This is an Open Access article distributed in accordance with the Creative Commons Attribution-NonCommercial-NoDerivs 4.0 International License (CC BY-NC-ND 4.0), which permits the noncommercial replication and distribution of the article with the strict proviso that no changes or edits are made and the original work is properly cited (including links to both the formal publication through the relevant DOI and the license). See: https://creativecommons.org/licenses/by-nc-nd/4.0/.

\section{References}

1. Freddo TF. A contemporary concept of the blood-aqueous barrier. Prog Retin Eye Res 2013;32:181-95.

2. Taylor AW. Ocular immunosuppressive microenvironment. Chem Immunol Allergy 2007;92:71-85.

3. Niederkorn JY. The induction of anterior chamberassociated immune deviation. Chem Immunol Allergy 2007;92:27-35.

4. Cone R, Chattopadhyay S, O'Rourke J. Control of delayed-type hypersensitivity by ocular- induced CD8+ regulatory t cells. Chem Immunol Allergy 2008;94:138-49.

5. Skelsey ME, Mayhew E, Niederkorn JY. CD25+, interleukin-10-producing CD4+ $\mathrm{T}$ cells are required for suppressor cell production and immune privilege in the anterior chamber of the eye. Immunology 2003;110:18-29.

6. Denniston AK, Kottoor SH, Khan I, et al. Endogenous cortisol and TGF-beta in human aqueous humor contribute to ocular immune privilege by regulating dendritic cell function. J Immunol 2011;186:305-11.

7. Paunicka K, Chen PW, Niederkorn JY. Role of IFNgamma in the establishment of anterior chamberassociated immune deviation (ACAID)-induced CD8+ T regulatory cells. J Leukoc Biol 2012;91:475-83. 
8. Chen W, Lin H, Zhong X, et al. Discrepant expression of cytokines in inflammation- and age-related cataract patients. PLoS One 2014;9:e109647.

9. Wu X, Liu Z, Wang D, et al. Preoperative profile of inflammatory factors in aqueous humor correlates with postoperative inflammatory response in patients with congenital cataract. Molecular Vision 2018;24:414-24.

10. Wu X, Long E, Lin H, et al. Prevalence and epidemiological characteristics of congenital cataract: a systematic review and meta-analysis. Sci Rep 2016;6:28564.

11. Plager DA, Lynn MJ, Buckley EG, et al. Complications in the first 5 years following cataract surgery in infants with and without intraocular lens implantation in the Infant Aphakia Treatment Study. Am J Ophthalmol 2014;158:892-8.

12. Plager DA, Lynn MJ, Buckley EG, et al. Complications, Adverse Events, and Additional Intraocular Surgery 1 Year After Cataract Surgery in the Infant Aphakia Treatment Study. Ophthalmology 2011;118:2330-4.

13. Plager DA, Yang S, Neely D, et al. Complications in the first year following cataract surgery with and without IOL in infants and older children. J AAPOS 2002;6:9-14.

14. Solebo AL, Russell-Eggitt I, Cumberland PM, et al. Risks and outcomes associated with primary intraocular lens implantation in children under 2 years of age: the IoLunder2 cohort study. Br J Ophthalmol 2015;99:1471-6.

15. Nixon DR, Woodcock MG. Pattern of posterior capsule opacification models 2 years postoperatively with 2 singlepiece acrylic intraocular lenses. J Cataract Refract Surg 2010;36:929-34.

16. Tetz MR, Auffarth GU, Sperker M, et al. Photographic image analysis system of posterior capsule opacification. J Cataract Refract Surg 1997;23:1515-20.

17. Friedman J, Hastie T, Tibshirani R. Regularization Paths for Generalized Linear Models via Coordinate Descent. J

Cite this article as: Lai $\mathrm{W}, \mathrm{Wu} \mathrm{X}$, Wang D, Liu Z, Lin D, Zhao L, Chen W, Lin H, Liu Y. Developmental characteristics of the cytokine profile in aqueous humor and its relationship with the inflammatory response in children. Ann Transl Med 2020;8(22):1542. doi: 10.21037/atm-19-2377
Stat Softw 2010;33:1-22.

18. Faul F, Erdfelder E, Lang AG, et al. G*Power 3: a flexible statistical power analysis program for the social, behavioral, and biomedical sciences. Behav Res Methods 2007;39:175-91.

19. Sijssens KM, Rijkers GT, Rothova A, et al. Distinct cytokine patterns in the aqueous humor of children, adolescents and adults with uveitis. Ocul Immunol Inflamm 2008;16:211-6.

20. Feng J, Zheng X, Li B, et al. Differences in aqueous concentrations of cytokines in paediatric and adult patients with Coats' disease. Acta Ophthalmologica 2017;95:608-12.

21. Zheng Y, Rao YQ, Li JK, et al. Age-related proinflammatory and pro-angiogenic changes in human aqueous humor. Int J Ophthalmol 2018;11:196-200.

22. Shehata HH, Abou Ghalia AH, Elsayed EK, et al. Clinical significance of high levels of survivin and transforming growth factor beta-1 proteins in aqueous humor and serum of retinoblastoma patients. J AAPOS 2016;20:444.e1-444.e9.

23. Cheng Y, Zheng S, Pan CT, et al. Analysis of aqueous humor concentrations of cytokines in retinoblastoma. PLoS One 2017;12:e0177337.

24. Cao Q, Xiao B, Jin G, et al. Expression of transforming growth factor $\beta$ and matrix metalloproteinases in the aqueous humor of patients with congenital ectopia lentis. Mol Med Rep 2019;20:559-66.

25. Zhang F, Chang P, Zhao Y, et al. A negative correlation of axial length with aqueous humor concentration of cytokines in patients with congenital cataracts. Mol Vis 2020;26:91-6.

26. Taub DD. Cytokine, growth factor, and chemokine ligand database. Curr Protoc Immunol 2004;Chapter 6:Unit 6.29.

27. Kawai M, Inoue $T$, Inatani $M$, et al. Elevated levels of monocyte chemoattractant protein-1 in the aqueous humor after phacoemulsification. Invest Ophthalmol Vis Sci 2012;53:7951-60. 